\title{
Emergency management of stroke in the era of mechanical thrombectomy
}

eISSN: 2383-4625

\section{Ethan S. Brandler, Nayeem Baksh}

Department of Emergency Medicine, State University of New York at Stony Brook, Stony Brook, NY, USA

Emergency management of stroke has been directed at the delivery of recombinant tissue plasminogen activator (TPA) in a timely fashion. Because of the many limitations attached to the delivery of tPA and the perceived benefits accrued to tPA, its use has been limited. Mechanical thrombectomy, a far superior therapy for the largest and most disabling strokes, large vessel occlusions (LVOs), has changed the way acute strokes are managed. Aside from the rush to deliver tPA, there is now a need to identify LVO and refer those patients with LVO to physicians and facilities capable of delivering urgent thrombectomy. Other parts of emergency department management of stroke are directed at identifying and mitigating risk factors for future strokes and at preventing further damage from occurring. We review here the most recent literature supporting these advances in stroke care and present a framework for understanding the role that emergency physicians play in acute stroke care.

Keywords Stroke; Large vessel occlusion; Emergencies; Tissue plasminogen activator; Mechanical thrombectomy

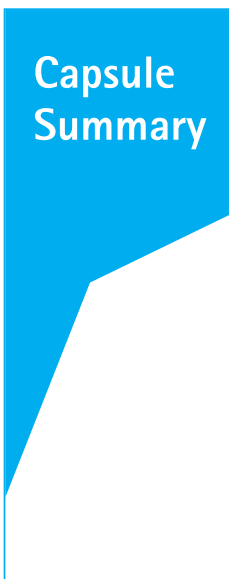

What is already known

Emergency management of stroke has been directed at the delivery of recombinant tissue plasminogen activator (TPA) in a timely fashion. Because of the many limitations attached to the delivery of tPA and the perceived benefits accrued to IPA, its use has been limited. Mechanical thrombectomy has changed the way acute strokes are managed. Other parts of emergency department management of stroke are directed at identifying and mitigating risk factors for future strokes and at preventing further damage from occurring.

What is new in the current study

We review the most recent literature supporting these advances in stroke care and present a framework for understanding the role that emergency physicians play in acute stroke care.
Received: 3 August 2018

Revised: 13 October 2018

Accepted: 24 October 2018

Correspondence to: Ethan S. Brandler Department of Emergency Medicine, State University of New York at Stony Brook, Health Sciences Tower, Level 4 Rm 080, Stony Brook, NY 11794-8350, USA

E-mail: ethan.brandler@ stonybrookmedicine.edu ORCID http://orcid.org/0000-0003-4647-8483

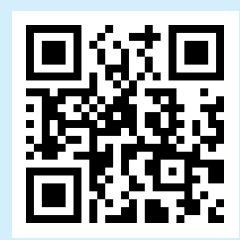

How to cite this article: Brandler ES, Baksh N. Emergency management of stroke in the era of mechanical thrombectomy. Clin Exp Emerg Med 2019;6(4):273-287.

This is an Open Access article distributed under the terms of the Creative Commons Attribution Non-Commercial License (http:// creativecommons.org/licenses/by-nc/4.0/). 


\section{INTRODUCTION}

Every year, 15 million people around the globe suffer a stroke leaving 5 million dead and another 5 million with permanent disability, and causing the loss of 113 million disability adjusted life years. ${ }^{1}$ This makes stroke the second leading cause of death worldwide behind ischemic heart disease. ${ }^{1,2}$

The impact of stroke is felt in the United States and across the world. There are significant racial differences in the epidemiology of stroke. ${ }^{3}$ Every 4 minutes someone dies of stroke and, every 40 seconds, someone in the United States suffers a stroke making stroke the number five leading cause of death in the United States. ${ }^{1}$ The vast majority, 87\%, of these strokes are ischemic. In contrast, in Korea where strokes occur every five minutes: only slightly less frequently, 24\% are hemorrhagic, resulting in 26,000 deaths annually. ${ }^{2}$ Within the United States population, stroke disproportionately affects African Americans more than any other racial group, with a death rate of 50.3/100,000 for African Americans as compared to $35.0 / 100,000$ for Caucasian Americans. ${ }^{1.4}$ The stroke incidence for populations greater than or equal to the age of 20 is 223/100,000 for African Americans and 93/100,000 for Whites. ${ }^{5}$ Older populations are affected more frequently by stroke. For example, for African American populations within the ages of 45 to 54 , the stroke incidence is $160.1 / 100,000$ while the stroke incidence of African Americans ages $\geq 85$ is $2,554.3 / 100,000{ }^{3}$ Overall, stroke is the number one leading cause of long-term disability in the United States. ${ }^{2}$ The largest disability and mortality burden of stroke is attributable to large vessel occlusion (LVO). The estimated fraction of LVOs is about 20\% of all strokes based on a Korean registry study. ${ }^{6}$ Approximately $1 / 3$ of acute ischemic strokes in the United States are due to LVO?

Considered in terms of economic burden, stroke accounts for approximately $0.27 \%$ of the gross domestic product spent on average by national health systems. ${ }^{3}$ Stroke causes a significant economic burden in Korea, costing an average 3,727 billion Korean won (3.3 billion US dollars). ${ }^{3}$ Stroke care costs an annual 34 billion US dollars within the United States, which includes the cost of health care services, medications, and missed days of work. 'In an effort to decrease the morbidity and mortality associated with stroke, prompt efficient and effective stroke care should be provided in the emergency setting.

\section{PREHOSPITAL CARE}

Emergent treatment of stroke begins in the prehospital setting with emergency medical services (EMSs), consisting of emergency medical technicians and paramedics. Stroke patients presenting with significant acute disability most often arrive by ambulance, which accounts for approximately 50\% of all stroke cases. The National Hospital Ambulatory Medical Care Survey found that out of 630,402 stroke patients in 2003 , only 331,760 arrived by ambulance. ${ }^{8}$ However, the percent of patients that arrive by ambulance varies between 38\% to 65\% of all stroke patients. ${ }^{9}$ Those with more severe strokes, hemorrhagic strokes, and older patients were more likely to utilize EMS. ${ }^{9}$ The use of EMS significantly improves stroke treatment and reduces the wait time for imaging and treatment, yet more than one third of stroke patients, mainly younger populations and minorities, fail to use EMS. ${ }^{10}$ More than half of all patients transported by EMS directly to definitive care hospitals arrived within two hours of symptom onset. ${ }^{11}$ In a Korean population of hemorrhagic stroke patients, the use of EMS markedly reduced the risk of mortality ${ }^{12}$ and was associated with decreased onset to admission times at definitive care hospitals. ${ }^{13}$ EMS should use a stroke screening tool to identify stroke patients in the prehospital setting. Part of this overall screening process should include screening for: stroke, stroke mimics, LVO, and for tissue plasminogen activator (tPA) contraindications. See Fig. 1 for a graphic depiction of the critical pathway for acute prehospital stroke care.

The clock for administering time sensitive therapies in acute stroke begins with the time of last known well. As postulated by Saver, ${ }^{14} 1.9$ million neurons are lost every minute in stroke until the offending vessel is opened. Delay in presentation to a hospital is one of the most significant factors in determining who will be eligible for treatment. ${ }^{10}$ Thus, one of the most important aspects of prehospital stroke screening is determining the last known well time: the time the patient was last seen to be at their normal baseline status. Additionally, glucose screening must be done rapidly to rule out the possibility of hypoglycemia masquerading as stroke. Stroke mimics are found to account for up to one third of suspected strokes. Todd's paralysis, migraine aura, hypoglycemia, and functional disorders are the most predominant conditions that lead to stroke mimics. ${ }^{15}$ Although no prehospital stroke screening tools is perfect, stroke screening in the prehospital setting must be done. Most strokes requiring treatment will be identified by one of the validated prehospital stroke scales. The use of a stroke assessment tool is recommended by the World Stroke Organization global stroke guidelines and action plan. ${ }^{16}$

Due to recent changes in acute endovascular care of LVO strokes, several LVO screening tools have been developed and utilized, including the Cincinnati Stroke Triage Assessment Tool and the Los Angeles Motor Scale (LAMS). The RACE (Rapid Arterial occlusion Examination) was also identified as a simple and effective tool for determining stroke severity in the prehospital setting, 


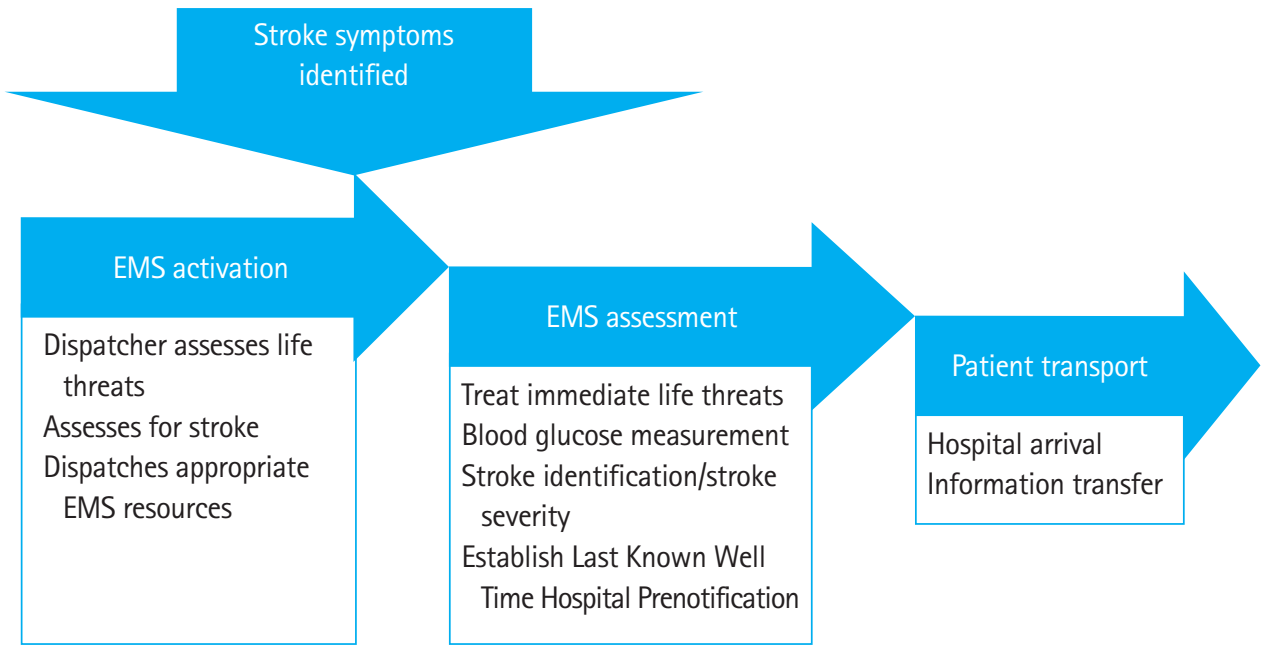

Fig. 1. Critical pathway for emergency medical service (EMS) management of suspected stroke.

and for identifying LVO. ${ }^{17}$ Although the LAMS and the Cincinnati Pre Hospital Stroke Screen were found to have similar diagnostic capabilities, LAMS was determined to be the most consistent screening tool because of its simplicity and universal application. ${ }^{18-21}$ Additionally, LAMS was found to outperform other prehospital stroke scales in terms of LVO prediction and was also more accurate than the National Institute of Health Stroke Scale (NIHSS) in the prehospital setting. ${ }^{22}$ The use of a prehospital stroke triage systems in order to efficiently direct stroke patients to primary stroke center in Chicago led to increased use of tPA. ${ }^{23}$ Additionally, the use of prehospital stroke screening tools to direct patients to comprehensive stroke centers will consequently reduce the number of patients at primary stroke centers, reduce the number of interhospital transfers, and reduce the time from symptom onset to groin puncture. ${ }^{24}$ The use of prehospital stroke triage systems, though imperfect, are clearly essentially for improving patient outcome, and thus demonstrates the necessity for urgent imaging. ${ }^{25}$

Stroke Emergency Mobile (STEMO) ambulances are equipped with a specialized stroke care team, a computed tomography (CT) scanner, and a point of care laboratory. The treatment of patients with cerebrovascular injury may be improved with the use of STEMO ambulances. ${ }^{26}$ STEMO was associated with a decrease in time to thrombolysis treatment as compared to traditional care, but more research is needed to confirm this. ${ }^{27}$ However, this may not be a cost-effective method of treating stroke patients. Furthermore, patients in isolated regions who may benefit the most from a STEMO ambulance most likely would not have access to this type of resource. ${ }^{28}$ Regardless of ambulance type, it is recommended that EMS prenotify the hospital of their estimated arrival time with the stroke patient. This notification should include the last known well time, the neurological deficits noted by the stroke screening or stroke severity scale used and report any conditions that might contraindicate the use of tPA.

\section{HOSPITAL ARRIVAL}

Emergent evaluation in the emergency department should focus on correction of immediate life threats and early image acquisition. Patients with functioning airway protective reflexes, normal oxygenation, normal or high blood pressure and normal blood glucose should proceed rapidly to the CT scanner or magnetic resonance imaging (MRI) as per local hospital protocol. If any of these are found to be abnormal, they should be corrected prior to scanning. A brief identification of neurological deficits by physical exam is essential, but there should not be any delays in obtaining initial imaging. While previous guidelines have recommended transporting patients directly from the ambulance to the CT scanner, there may be little added benefit, as this practice was found to have no change in reducing time to stroke treatment. ${ }^{29}$ Lab work is not indicated before a CT scan is completed but should include baseline electrolyte, hematologic and renal panels and troponins to look for concomitant Ml of cardiac strain. Metaanalysis concludes that CT angiography (CTA) or CT perfusion (CTP) did not increase the risk of kidney injury in stroke patients, or even patients with known kidney disease, thus performing CT before lab work is acceptable. ${ }^{30}$ Complete NIHSS stroke scale, a detailed history, and physical should follow imaging or occur during imaging interpretation by radiology. The last known well time must be determined, and family or friends should be contacted accordingly, potentially while the patient is undergoing a CT scan. See Fig. 2 for a graphic depiction of the critical pathway for acute 


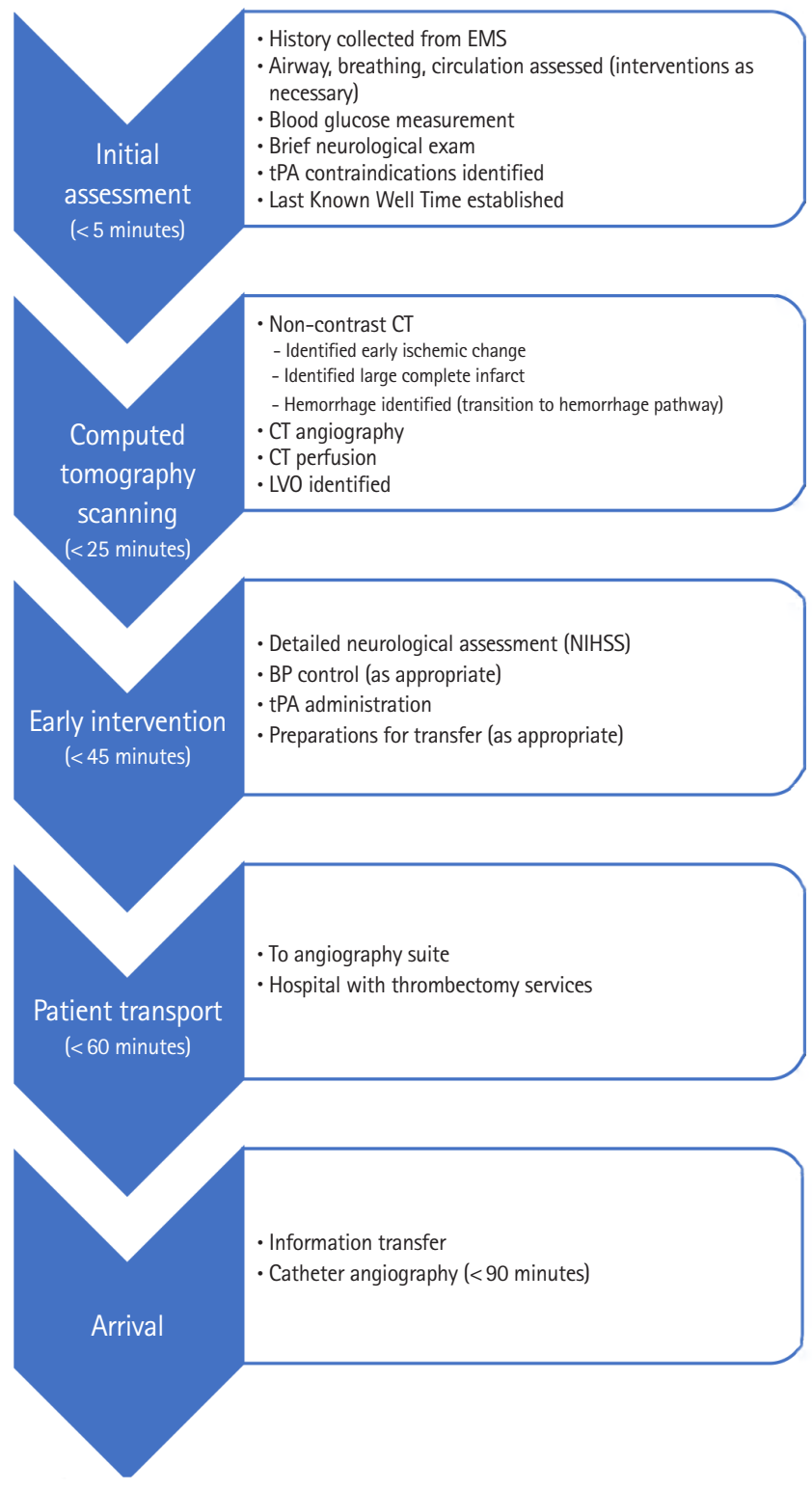

Fig. 2. Emergency Department Clinical Pathway.

EMS, emergency medical system; CT, computed tomography; LVO, large vessel occlusion; NIHSS, National Institutes of Health Stroke Scale; BP, blood pressure; tPA, tissue plasminogen activator.

emergency department stroke care.

Stroke severity tools are critical in the hospital setting. Severity screening is important for several reasons. First, it offers providers across disciplines a way to evaluate and follow a stroke patient's care course objectively. Second, severity may determine the need for additional therapies such as endovascular thrombectomy or hemi-craniotomy. Thirdly, patients with rapidly improving or minor symptoms may or may not benefit from treatment. The Potential of rtPA for Ischemic Strokes With Mild Symptoms (PRISMS) trial, though curtailed, provides evidence that patients without obvious debilitating deficits do not benefit from tPA treatment. ${ }^{31}$ Additional research is needed to confirm this finding. The most widely accepted stroke severity scale in the hospital setting is the NIHSS scale. A complete copy of the NIHSS scale can be found in Table 4 of the 2018 stroke guidelines. ${ }^{32}$ The simplicity of this test makes it the best candidate for in-hospital stroke severity screening. ${ }^{33,34}$ However, the NIHSS is not a perfect scale, but it is the best option available currently. Scoring a zero on the NIHSS test does not always equate to an absence of stroke. ${ }^{35}$ No stroke scale was found to accurately predict LVO with high sensitivity and specificity. Any clinical exam based LVO screening tool will inevitably miss some patients that do have LVO, as well as some patients with milder strokes..$^{25,36}$

Because one in five patients with ischemic stroke or transient ischemic attack (TIA) have a history of atrial fibrillation or are found to have atrial fibrillation on their initial 12 lead electrocardiogram when they present with ischemic stroke, patients should have cardiac rhythm monitoring as soon as possible after presentation and for the duration of the hospital stay. ${ }^{37}$ The identification of atrial fibrillation provides a target for secondary prevention in the form of anticoagulation medications.

\section{IMAGING}

Non-contrast CT scanning has been the Initial imaging test of choice in acute stroke care. While the initial non-contrast CT does little to elucidate the extent or location of a very acute stroke, non-contrast CT nevertheless rules out acute hemorrhage and large masses that exclude the possibility of thrombolytic treatment and may suggest alternative diagnoses.

Furthermore, hemorrhagic and ischemic strokes are managed quite differently. Once a stroke is determined to be ischemic, it is necessary to determine the presence or absence of a LVO that is amenable to endovascular care. CT may also detect subtle changes in the parenchyma and suggest the possibility of a LVO.

The Alberta Stroke Program Early CT Score, performed on a non-contrast $\mathrm{CT}$, represents the volume of irreversibly damaged tissue, and can be used in conjunction with the CTA collateral score to improve outcome prediction of patients. ${ }^{38}$ MRI may be better for imaging stroke patients in some instances. When comparing MRI versus $\mathrm{CT}$ imaging, MRI was found to be more sensitive in determining ischemic stroke than was CT imaging. Changes in acute ischemic stroke can be seen more easily and earlier with MRI, and diagnosis reliability is greater with MRI. ${ }^{39}$ Nonetheless, CT was determined to be the better option, since CT scans are more rapidly conducted, and more cost effective. ${ }^{40}$

However, the unreliability of clinical stroke scales increases the 
importance of advanced imaging both of the blood vessels and the parenchyma. For example, $9 \%$ of patients with a NIHSS score of 4 of less were found to have an LVO on vascular imaging, demonstrating the unreliability of clinical stroke scales. ${ }^{41}$ There are several different types of imaging techniques, each having their pros and cons. CT and MRI are both effective methods for imaging stroke patients' blood vessels and brain parenchyma. Both have been shown to be useful in determining eligibility for thrombolytic and endovascular mechanical therapies. ${ }^{42-44}$ This information is critical for establishing a safe, effective treatment plan.

Advanced imaging with $\mathrm{CT}$ has several parts: the non-contrast CT, CTA, and CTP. CTA demonstrates the presence of a clot in a large vessel, the presence of an arterial dissection and can be used to show which areas of the brain are at risk for infarction and have collateral circulation. CTP can help to determine if there is an area of the brain at risk, ischemic but not yet infarcted. A combination of CTA and CTP imaging is the most widely used modality for determining eligibility for endovascular care though many use non-contrast CT and CTA only. CTA alone has greater diagnostic capability that CTP alone. . $^{4,46}$

When considering imaging choice, it is important to consider which method is quicker, thus allowing treatment to proceed with less delay. The ultimate goal is to reduce the picture to puncture time (P2P). Each hospital system will have their preferred method of imaging, which is acceptable, as long as there is no delay in PAA administration, and the overall P2P time is relatively low. As staff experience with one particular form of imaging increases, the P2P time will consequently decrease, which results in more favorable clinical outcomes for acute ischemic stroke patients. ${ }^{47}$ The World Stroke Organization supports the use of either CT scan or MRI, depending on which is more accessible and can be conducted rapidly. ${ }^{16}$

\section{BLOOD PRESSURE CONSIDERATIONS}

Blood pressures may be widely variable in stroke, and there is a U-shaped mortality curve associated with excessively low or high blood pressures. ${ }^{48}$ Very high blood pressures may indicate that a patient's cardiovascular system is attempting to autoregulate flow in a narrowed artery or arteries in an ischemic area of the brain. Treating that elevated blood pressure may result in decreased blood flow around stenotic lesions. As a result, it may be difficult to determine in which patients' blood pressure should be lowered acutely and in which patients' blood pressure should be allowed to remain high. Monitoring and treating an acute ischemic stroke patient's blood pressure is an intricate and potentially hazardous situation.

Because elevated blood pressure is thought to be a risk factor for subsequent hemorrhage, patients in whom thrombolytics is planned should have their blood pressure lowered to safe levels as per National Institute of Neurological Disorders and Stroke rtPA (NINDS) criteria. More detailed criteria regarding blood pressure can be found within the 2018 stroke guidelines..$^{32}$ In patients eligible for tPA treatment, systolic blood pressure should be lowered to less than $185 \mathrm{mmHg}$ and diastolic blood pressure should be lowered to less than $110 \mathrm{mmHg}$ before tPA treatment is initiated.

In patient's not receiving tPA, the benefits of lowering blood pressure are less clear. Decreasing blood pressure too drastically may exacerbate cerebral ischemia. At this time, there is insufficient evidence that automatically lowering blood pressure is beneficial or may reduce mortality. Antihypertensive treatment as secondary stroke prevention in patients not receiving acute stroke treatment, should only be given once the patient is medically and neurologically stable.

Patients for whom endovascular and no thrombolytic treatments are planned should not have their autoregulation of blood pressure disturbed until the occlusion is cleared in the angiography suite. ${ }^{49,50}$ Overzealous blood pressure control in this situation may reduce collateral blood flow across a stenotic lesion. Blood pressure may be lowered after vessel opening. Further research is needed to determine the effects of automatically lowering blood pressure in ischemic stroke patients. ${ }^{51}$ It is generally accepted than lowering excessive systolic blood pressure ( $>220 \mathrm{mmHg}$ ) is safe and recommended. ${ }^{52}$ Lower blood pressure in the first 24 hours post stroke was associated with reduced risks of intracerebral hemorrhage. ${ }^{53}$ For patients who are critically ill or are to receive TPA or other interventions, clevidipine and nicardipine are the agents of choice for rapidly lowering blood pressure. ${ }^{32,54}$

Rapid fluctuations in blood pressure within the first $180 \mathrm{~min}$ utes of hospital stay were associated with increased mortality rate within 90 days for acute ischemic stroke patients. As compared to patients with smaller variations in blood pressure, patients who died within 90 days were found to have larger changes in their diastolic blood pressures within the first 180 minutes. $^{55}$ A rapid spontaneous drop in systolic blood pressure may indicate that recanalization has occurred.

\section{THROMBOLYTIC THERAPY}

Until the 1995 publication of the seminal NINDS tPA trial, stroke treatment was limited to supportive care. Since that time, tPA has been demonstrated to be an effective therapy with varied interpretation of reported trials and observational studies. Of the many thrombolytic therapies that have been suggested for the 
treatment of ischemic stroke, only tPA (recombinant tPA [alteplase] and its cousin tenecteplase [TNKase]) have been shown to be useful and will be discussed here. ${ }^{56,57}$ tPA effectiveness and perhaps safety decline with the passage of time from ictus, thus determining the last known well time of the patient is critical. A recent review of major trials of tPA, including NINDS and European Cooperative Acute Stroke Study III (ECASS III), suggests that the majority of patients presenting within 4.5 hours should receive tPA treatment unless there is a contraindication. ${ }^{58}$ According to the NINDS trial, tPA should be given to patients as soon as possible, ideally within 90 minutes. There have been benefits to treating patients with IPA after 3 hours, but with increased risks. Treatment with tPA within 3 hours was associated with improved clinical outcomes assessed at three-month post follow up..$^{59-61}$ The time frame for IPA treatment is critical, and there is support for the use of tPA up to 4.5 hours after the onset of stroke symptoms. ${ }^{62}$ Although a slightly higher risk of intracerebral hemorrhage was associated with IPA treatment, meta-analysis determined this risk is minimal and that IPA is a safe and effective treatment. ${ }^{63}$ Extremely careful consideration should be given prior to administering IPA in patients with contraindications to thrombolytic therapy such as: past or present intracranial hemorrhage, severe uncontrolled hypertension, severe hypoglycemia/hyperglycemia, serious head trauma or stroke within the past 3 months, thrombocytopenia, coagulopathies, including those associated with the use of anticoagulant medications, low molecular weight heparins, direct thrombin inhibitors, and factor Xa inhibitors. ${ }^{64,65}$ The risk of administering tPA must be considered versus the benefit prior to treatment.

Rapid expert consultation may be helpful in selecting patients for thrombolytic therapy and for possible endovascular care. In hospitals where a vascular neurologist may not be immediately available, this consultation can come in the form of a telemedicine consult with a remote expert. ${ }^{66}$

Outcomes in modern stroke treatment trials are based on the 90-day modified Rankin Score. A good outcome is described as a 0 or 1 on the modified Rankin Scale (mRS) indicating little (1) or (0) no disability. An mRS of 6 describes death. Some trials include an mRS of 2 (slight disability) as a good outcome. ${ }^{67}$ For any treatment, the number needed to treat to achieve a good outcome on $\mathrm{mRS}$ is a valuable tool in assessing the overall safety and efficacy of that particular treatment. For IPA, the number needed to treat increases as time from symptom onset progresses. The sooner IPA is given, the greater the benefit appears to be, with reduced associated risk. ${ }^{59}$ For example, one study determined that out of 100 patients, 27.8 benefited from tPA treatment within 90 minutes, and 1.5 were harmed. However, as the time from symptom onset increases to between 271 and 360 minutes, 5.2 patients benefited while 7.3 were harmed. ${ }^{68} \mathrm{~A}$ review comparing the NINDS trial with the ECASS trial found that within 3 hours of tPA treatment, 32.3 patients out of 100 benefited while 3.3 were harmed. Within 3 to 4.5 hours, 16.4 patients out of 100 benefited, while 2.7 were harmed. ${ }^{69}$ This demonstrates that tPA treatment is extremely time sensitive and can have pernicious effects as time from symptom onset increases. However, two recent trials have suggested that time is not the most important variable and that ability to withstand prolonged ischemia before revascularization is dependent on other factors such as collateral blood flow. Newer imaging techniques may be able to separate those more likely to benefit from those that will not. ${ }^{70,71}$

Aside from time, the size of the clot and its location may be determining factors in treatment effectiveness. The number needed to treat for IPA treatment benefit in LVO may be even higher than for occlusions of smaller vessels. tPA treatment alone was associated with lower overall functional independence at 90 days as compared to combined treatment of IPA with endovascular reperfusion. ${ }^{72}$ The NINDS trial showed that tPA has beneficial value, but only around 32 out of 100 patients benefit, and only 13 out of 100 end up functionally normal after the treatment. Three out of the 100 patients ended up worse after the treatment. This data shows the urgent necessity for better treatment options. ${ }^{73}$ The value of tPA for mild strokes (NIHSS less than or equal to 5) needs to be further researched to test for efficacy and patient safety ${ }^{74}$ but the prematurely closed PRISMS trial showed little benefit to treatment of very mild strokes with tPA. $^{31,75}$

Newer studies have shown that tenecteplase is just as effective, if not more so, than tPA. Meta-analysis of three studies concluded that tenecteplase was effective and safe for use. When comparing tenecteplase to IPA, some research suggests that there is no difference in patient outcome with either option..$^{56,76}$ Furthermore, a recent sub-group analysis of the Tenecteplase versus Alteplase before Endovascular Therapy for Ischemic Stroke (EXTEND-IA TNK) trial suggests that tenecteplase is more effective in opening occluded vessels than tPA. ${ }^{77}$ An important feature of tenecteplase is its ease of use. Tenecteplase can be administered more rapidly, as an intravenous bolus, to ischemic stroke patients without an extended administration via intravenous drip. Thus, dosing calculations and administration are much simpler and emergent transport between primary and comprehensive centers is easier without an ongoing tPA infusion.

In the EXTEND-IA TNK trial, tenecteplase produced better outcomes as compared to standard dosing of intravenous alteplase. Recanalization after thrombolytic therapy was markedly higher in 
those that received tenecteplase as compared to alteplase. There is no significant difference in the risk of cerebral ischemic hemorrhage between the two treatments, and therefore tenecteplase should be considered just as effective and safe, if not better, than alteplase. $^{77}$

\section{ENDOVASCULAR THERAPY}

The most effective therapy for stroke is endovascular thrombectomy. For patients with LVOs evident on CTA or magnetic resonance angiography, the number needed to treat may be as low as 2.45. In 2015, several major trials were presented showing the unparalleled benefits of endovascular thrombectomy. With any stroke treatment plan, treatment should begin as quickly as possible, which applies to endovascular care as well. Thrombectomy with a stent retriever within 6 hours of symptom onset improved overall patient outcome at 90 days post stroke. ${ }^{78}$ Moreover, patient outcome at 90 days was shown to be better in patients who received thrombectomy in addition to standard care, as opposed to standard care alone. ${ }^{79}$ The efficacy and safety of mechanical thrombectomy for the treatment of ischemic stroke was demonstrated by seven major trials published in late 2014 and 2015: Multicenter Randomized Clinical Trial of Endovascular Treatment for Acute Ischemic Stroke in the Netherlands (MR CLEAN), ${ }^{80}$ EXTEND-IA ${ }_{1}^{81}$ REVASCAT (Randomized Trial of Revascularization with Solitaire FR Device versus Best Medical Therapy in the Treatment of Acute Stroke Due to Anterior Circulation Large Vessel Occlusion Presenting within Eight Hours of Symptom Onset), ${ }_{1}^{82}$ REVAS$\mathrm{CAT}_{1}^{82}$ SWIFTPRIME (Solitaire with the Intention for Thrombectomy as Primary Endovascular Treatment), ${ }^{78}$ ESCAPE (Endovascular Treatment for Small Core and Anterior Circulation Proximal Occlusion with Emphasis on Minimizing CT to Recanalization Times), ${ }_{1}^{83,84}$ THRACE, ${ }^{85}$ and THERAPY (Assess the Penumbra System in the Treatment of Acute Stroke). ${ }^{86}$ The trials provide overwhelming evidence that endovascular mechanical thrombectomy is safe, effective, and improves functional outcomes across a broad range of patient demographics and stroke severities. Approximately $70 \%$ of thrombectomy patients in these trials received tPA before endovascular treatment and a marginal benefit was noted to the combined treatment as compared to thrombectomy alone. ${ }^{87}$ The DEFUSE (Endovascular Therapy Following Imaging Evaluation for Ischemic Stroke) 3 trial demonstrated that mechanical thrombectomy may be a safe treatment for ischemic stroke up to 16 hours after the last known well time, while the DAWN (DWI or CTP Assessment with Clinical Mismatch in the Triage of Wake-Up and Late Presenting Strokes Undergoing Neurointervention with Trevo) trial shows that thrombectomy may be safe up to 24 hours past the last known well time, and thrombectomy in patients with favorable findings on perfusion imaging was associated with improved functional outcomes. ${ }^{79,88}$

Because longer P2P times are associated with less favorable patient functional outcomes, ${ }^{89}$ patients with suspected LVO should be transported to a facility equipped and staffed to perform endovascular thrombectomy in a rapid fashion. This process ideally begins in the field where patients with physical exams suggesting LVO are transported preferentially to such centers, however, since physical exam is not sensitive enough to identify all LVOs, vessel imaging should be performed early in the patient's emergency department visit. In facilities without access to high quality angiography, NIHSS severity thresholds may be used to identify those patients most likely to have a LVO.

\section{PATIENT TRANSFER}

Optimal care of stroke patients is limited by the availability of experts and equipment. Transfer to a more capable center should be considered when a stroke patient's needs exceed the capability of the receiving hospital. Community hospitals may be able to deliver the appropriate diagnostic imaging and tPA but may not be able to deliver advanced interventions such as endovascular therapy. The availability of expert advice from stroke neurologists may be improved by the use of telemedicine. A telemedicine-based neurologist may be consulted when planning for acute treatment and may advise on the utility and appropriateness of transfer. Further development of telestroke networks may lead to improved care for ischemic stroke patients. ${ }^{90}$ Thus far, the increased use of telestroke networks has increased the number of patients transported after tPA for more advanced care. ${ }^{66}$ However, because time is critical when treating a stroke patient, any unnecessary delay in hospital transfer is associated with worse patient outcomes. ${ }^{91}$ Prearranged transfer agreements and transfer plans are needed to facilitate the process. Timely communication between sending and receiving facilities is essential to decrease the time to revascularization and to improve patient outcomes. ${ }^{66}$ Computerized transfer of images and clinical information further facilitates this process. ${ }^{66}$

Delays in interhospital transfers are one of the main reasons why acute ischemic stroke patients are excluded from tPA treatment. ${ }^{92}$ Interhospital transfer may also result in worse outcomes for patients initially appropriate to undergo thrombectomy. ${ }^{93}$ For patients undergoing mechanical thrombectomy, stroke onset to revascularization time for patients transported directly to endovascular-capable centers was significantly shorter as compared to interhospital transfer. ${ }^{93}$ The P2P times for interhospital transfer 
patients were longer on average, as compared to patients who were directly transported. ${ }^{89}$ Furthermore, a Madrid study found that $41 \%$ of interhospital transfer patients were futile, possibly due to delayed revascularization associated with interhospital transfer. ${ }^{89,94}$

\section{TIA/MINOR STROKE}

For patients presenting with either TIA or milder strokes, there is no required emergent treatment. Focus in these cases is directed at secondary prevention. Focus in these cases is directed at secondary prevention and risk factor modification. Selected patients may be discharged after a brief emergency department visit or short hospital stay provided that adequate and urgent follow up is available. The ABCD2 score and the more refined ABCD3-I score ${ }^{95}$ may be used to determine short term stroke risk and thus the need for hospitalization. ${ }^{96}$ For the secondary prevention of TIA or stroke, the combination of clopidogrel and aspirin is slightly better than the use of aspirin alone. ${ }^{97-99}$ Attention must be paid to aggressive control of diabetes, hypertension and hyperlipidemia where they are identified. ${ }^{100}$

\section{ATRIAL FIBRILLATION}

Many patients with stroke are noted to have atrial fibrillation on presentation. However, for many patients, paroxysmal atrial fibrillation may not have been previously identified or may not be present at time of evaluation. Secondary prevention also has to focus on a search for atrial fibrillation because up to thirty percent of patients presenting with "cryptogenic" stroke may in fact have paroxysmal atrial fibrillation. ${ }^{101}$ Patients with atrial fibrillation related strokes may be treated with long term anticoagulation therapy to prevent stroke recurrence. CHA2DS2-VASc scoring may be used to assess the risk of stroke in these patients, but this score was developed to balance the benefits and risks of vitamin $\mathrm{K}$ antagonists. ${ }^{102}$ The risk of bleeding in patients taking NOACs (Novel oral anti-coagluants) is thought to be better with a marginal improvement in stroke outcomes as compared to vitamin $\mathrm{K}$ antagonist. ${ }^{103}$ The CHA2DS2-VASc score was associated with the risk of ischemic stroke, thromboembolism, and death. The predictive ability of this scoring system was limited, however. ${ }^{104}$ In patients in whom anticoagulation is contraindicated, consideration of mechanical devices should be considered. ${ }^{105}$ The HAVOC score may be used to identify patients in sinus rhythm at the time of evaluation at risk for atrial fibrillation related stroke and for possible long term cardiac rhythm monitoring. ${ }^{106}$

\section{CRYPTOGENIC STROKE}

Thirty percent of cryptogenic strokes are the result of silent and paroxysmal atrial fibrillation. Some other causes of cryptogenic stroke are: cardiac embolism, vasculopathy (most commonly aortic plaques and Fabry's disease), and coagulopathy. ${ }^{107}$ The use of tobacco may be linked to cryptogenic stroke in young adults. ${ }^{108}$ The remainder of cryptogenic strokes are caused by patent foramen ovale or hypercoagulable states. ${ }^{101}$ In patients who are found to have patent foramen ovale, patent foramen ovale-occluding devices may decrease the risk of recurrent stroke compared with medical therapy in patients with cryptogenic stroke. ${ }^{109}$ There are clear conditions that increase the likelihood of a patient having recurrent strokes. No predictive scale may be 100\% accurate, however, it is still critical to determine which patients may be at greater risk and change treatment plans accordingly. It is unclear if echocardiography should be recommended for patients with cryptogenic stroke. ${ }^{110}$ Echocardiography may be beneficial for certain patients, yet unnecessary for others. ${ }^{111}$ In younger patients or those with a suspected paraneoplastic syndrome, a search for hypercoagulable states might be undertaken. ${ }^{112,113}$

\section{AIRWAY/DYSPHAGIA SCREENING}

Maintenance of an unobstructed airway is critical and must be monitored in post stroke patients. Dysphagia is common in several stroke patients and can lead to several complications. All stroke patients should be screened for dysphagia. However, one in five patients with acute ischemic stroke were not subjected to dysphagia screening, and patients with mild stroke or TIA were even less likely to undergo dysphagia screening. Failing dysphagia screening is associated with poorer patient outcomes. ${ }^{114}$ There are several other tests that can be used for dysphagia as well. It is vital that all stroke patients be screened for dysphagia. Patients who suffered a stroke are also at risk for pneumonia. The cough reflex test-used to test for dysphagia-was not correlated with reducing the risk of pneumonia later on. ${ }^{115}$ However, additional research has shown that a swallow test with subsequent modifications in consistency of diet administered by a nurse and speech pathologist has led to decreased rates of pneumonia among patients with stroke. ${ }^{116}$ The Gugging Swallowing Screen method and intensive oral hygiene care were associated with lower rates of post stroke pneumonia. ${ }^{117}$ The use of antibiotics for post stroke prevention of pneumonia is not recommended if the patient has dysphagia. ${ }^{118}$ 


\section{POST STROKE REHAB}

Though not typically the role of the emergency physician, it is important for the emergency physician to understand that suffering a stroke is a financially, physically, and emotionally straining experience. Depending on the location and severity of the stroke, certain bodily functions may be permanently altered. After suffering a stroke, many patients become depressed. Depression screening should be conducted on all patients who suffer a stroke. There are several different scales to determine if a patient is depressed, yet none are perfect. ${ }^{119}$ Physical rehabilitation may be needed in certain patients who have decreased motor function post stroke. Both strength training and stretching are proven to improve functional activity, and correct postural asymmetry for patients who previously suffered a stroke. ${ }^{120}$ Additionally, with the vast prevalence of technology in the modern world, a new study is focused on determining if the use of touchscreen tablets can help stroke patients increase their dexterity in their affected limb. ${ }^{121}$ Sexologists and sex therapists are recommended to be part of the post- stroke care team, as sexual function can decrease drastically in stroke patients. This decrease in sexual function can also contribute to the patient's depressive mood. ${ }^{122}$ Post stroke rehab is an extensive process that may or may not improve patient function. Some factors to consider when determining what rehab methods are optimal are: stroke severity and location, patient baseline, and patient age.

\section{DEEP VEIN THROMBOSIS PROPHYLAXIS}

Deep vein thrombosis (DVT) is a common post stroke complication. The incidence of DVT in stroke patients is between 10\% to $75 \%$ and this variation is due to differences in diagnostic method and time of evaluation. ${ }^{123}$ There are several methods to prevent this occurrence. One method, intermittent pneumatic compression, involves a machine compression system. This is regarded as the best overall method to reduce the probability of DVT. Some other methods involve prophylactic anticoagulation with unfractionated heparin, low molecular weight heparins, and neuromuscular electrical stimulation. Of all the methods previously shown, intermittent pneumatic compression has demonstrated the best outcomes. ${ }^{124-126}$

\section{HEMORRHAGIC STROKE}

Though hemorrhagic strokes constitute only $15 \%$ of all stroke, the management of acute hemorrhagic stroke is intuitively different than the management of acute ischemic stroke. Early imaging plays a crucial role in differentiating between ischemic and hemorrhagic stroke. Treatment of hemorrhage is directed at limiting extension of the bleeding. Blood pressure should be controlled to less than $180 \mathrm{mmHg}$, and possibly as low as $140 \mathrm{mmHg}$ different from ischemic stroke patients. ${ }^{177}$ The ATACH2 (Antihypertensive Treatment of Acute Cerebral Hemorrhage II) and ACT2 (Inter-intensive Blood Pressure Reduction in Acute Cerebral Hemorrhage Trial II) trials demonstrated that intensively lowering blood pressure in hemorrhagic stroke patients may not decrease mortality rate or the rate of disability. ${ }^{128,129}$ However, because in these trials the actual blood pressure was closer to $140 \mathrm{mmHg}$, many clinicians have interpreted these studies as showing a benefit of having a lower blood pressure and recent guidelines ${ }^{127}$ reflect a blood pressure closer to $140 \mathrm{mmHg}$ systolic. Prothrombin complex concentrates, the activated prothrombin complex concentrate FEIBA (factor VIII inhibitor bypassing activity), recombinant activated factor VIla, and vitamin $\mathrm{K}$ have emerged as potential therapies for bleeding related to anticoagulants ${ }^{127,130}$ but may be harmful in those not previously on anticoagulation. Specifically, recombinant activated factor VIla is not recommended as there is an increased thrombotic risk associated with this treatment. The efficacy and safety associated with platelet transfusion therapy is undetermined for bleeding related to aspirin or thienopyridines. After bleeding has stopped, low molecular weight heparin or unfractionated heparin can be used to prevent thromboembolism. Drug specific antidotes (idracuzimab) to anticoagulants have been developed and rapidly approved for use, but the evidence for these is limited. ${ }^{131}$ Surgical removal of the hematoma is of limited use. Newer, less invasive therapies may improve outcomes. ${ }^{132}$

\section{ANTITHROMBOTICS}

Antithrombotics can be used to reduce the risk of stroke in patients diagnosed with atrial fibrillation. As previously mentioned, this can be a contraindication if the patient develops a hemorrhagic stroke. Antithrombotics given within 24 hours of recanalization therapy was not found to increase hemorrhage in patients, and thus may be beneficial. ${ }^{133}$ There are several different antithrombotic treatments that can potentially be used for stroke prevention. In patients taking Warfarin, the increased time-intherapeutic range corresponded with a decreased risk of stroke. Rivaroxaban, dabigatran, and apixaban are alternative treatments that are comparable to warfarin. ${ }^{134-136}$ Treatment with warfarin was determined to be more effective than antiplatelet treatment for reducing the risk of stroke. Furthermore, the absolute risk of hemorrhage associated with antithrombotic therapy was less than the absolute reduction in stroke. ${ }^{137,138}$ 


\section{CONCLUSION}

Efficient and effective stroke care begins in the ambulance with timely identification of strokes, triage of possible LVOs to endovascular capable centers and correction of hypoglycemia. Emergency department care is focused on early imaging to rule out hemorrhage and to identify LVO. Stroke specific therapy should be delivered as rapidly as possible while adhering to safe administration practices. Transfer for advanced therapies should be undertaken in a prearranged fashion as expeditiously as possible. Stroke mortality and morbidity can be reduced with the appropriate in-hospital supportive therapies and secondary prevention.

\section{CONFLICT OF INTEREST}

Ethan S. Brandler is supported by the Northeast Cerebrovascular Consortium for his research in prehospital stroke care. Except for that, no potential conflict of interest relevant to this article was reported.

\section{REFERENCES}

1. The Internet Stroke Center. Stroke statistics [Internet]. Dallas, TX: The Internet Stroke Center [cited 2018 May 3]. Available from: http://www.strokecenter.org/patients/about-stroke/ stroke-statistics/.

2. Hong KS, Bang OY, Kang DW, et al. Stroke statistics in Korea: part I. Epidemiology and risk factors: a report from the korean stroke society and clinical research center for stroke. J Stroke 2013;15:2-20.

3. Wang $Y$, Rudd AG, Wolfe $C D$. Age and ethnic disparities in incidence of stroke over time: the South London Stroke Register. Stroke 2013;44:3298-304.

4. US Department of Health and Human Services Office of Minority Health. Stoke and African Americans [Internet]. Rockville, MD: Office of Minority Health Resource Center; 2016 [cited 2018 May 13]. Available from: https://minorityhealth. hhs.gov/omh/browse.aspx? $|v|=4$ Ct|vlid $=28$.

5. Sacco RL, Boden-Albala B, Gan R, et al. Stroke incidence among white, black, and Hispanic residents of an urban community: the Northern Manhattan Stroke Study. Am J Epidemiol 1998; 147:259-68.

6. Kim S, Shin SD, Ro YS, et al. Effect of emergency medical services use on hospital outcomes of acute hemorrhagic stroke. Prehosp Emerg Care 2016;20:324-32.

7. Malhotra K, Gornbein J, Saver JL. Ischemic strokes due to large-vessel occlusions contribute disproportionately to stroke- related dependence and death: a review. Front Neurol 2017; 8:651.

8. Mohammad YM. Mode of arrival to the emergency department of stroke patients in the United States. J Vasc Interv Neurol 2008;1:83-6.

9. Adeoye 0 , Lindsell C, Broderick J, et al. Emergency medical services use by stroke patients: a population-based study. Am J Emerg Med 2009;27:141-5.

10. Ekundayo 0J, Saver JL, Fonarow GC, et al. Patterns of emergency medical services use and its association with timely stroke treatment: findings from Get With the GuidelinesStroke. Circ Cardiovasc Qual Outcomes 2013;6:262-9.

11. Park HA, Ahn KO, Shin SD, Cha WC, Ro YS. The effect of emergency medical service use and inter-hospital transfer on prehospital delay among ischemic stroke patients: a multicenter observational study. J Korean Med Sci 2016;31:139-46.

12. Hong $K S$, Ko SB, Yu KH, et al. Update of the Korean clinical practice guidelines for endovascular recanalization therapy in patients with acute ischemic stroke. J Stroke 2016;18: 102-13.

13. Kim DG, Kim YJ, Shin SD, et al. Effect of emergency medical service use on time interval from symptom onset to hospital admission for definitive care among patients with intracerebral hemorrhage: a multicenter observational study. Clin Exp Emerg Med 2017;4:168-77.

14. Saver JL. Time is brain: quantified. Stroke 2006;37:263-6.

15. Daniere $F_{1}$ Edjlali-Goujon M, Mellerio $C_{1}$ et al. MR screening of candidates for thrombolysis: How to identify stroke mimics? J Neuroradiol 2014;41:283-95.

16. Lindsay MP, Norrving $B$, Furie $K L$, Donnan G, Langhorne $P$, Davis $S$. Global stroke guidelines and action plan: a road map for quality stroke care. Geneva: World Stroke Organization; 2016.

17. Perez de la Ossa N, Carrera D, Gorchs M, et al. Design and validation of a prehospital stroke scale to predict large arterial occlusion: the rapid arterial occlusion evaluation scale. Stroke 2014:45:87-91.

18. Brandler ES, Sharma M, Sinert RH, Levine SR. Prehospital stroke scales in urban environments: a systematic review. Neurology 2014;82:2241-9.

19. McMullan JT, Katz B, Broderick J, Schmit P, Sucharew H, Adeoye 0 . Prospective prehospital evaluation of the cincinnati stroke triage assessment tool. Prehosp Emerg Care 2017;21: 481-8.

20. Llanes JN, Kidwell CS, Starkman S, Leary MC, Eckstein M, Saver JL. The Los Angeles Motor Scale (LAMS): a new measure to characterize stroke severity in the field. Prehosp Emerg 
Care 2004;8:46-50.

21. Jayaraman $M V$, lqbal $A$, Silver $B$, et al. Developing a statewide protocol to ensure patients with suspected emergent large vessel occlusion are directly triaged in the field to a comprehensive stroke center: how we did it. J Neurointerv Surg 2017;9:330-2.

22. Noorian AR, Sanossian N, Shkirkova K, et al. Los angeles motor scale to identify large vessel occlusion: prehospital validation and comparison with other screens. Stroke 2018;49: 565-72.

23. Prabhakaran S, O'Neill K, Stein-Spencer L, Walter J, Alberts MJ. Prehospital triage to primary stroke centers and rate of stroke thrombolysis. JAMA Neurol 2013;70:1126-32.

24. Schlemm L, Ebinger $\mathrm{M}$, Nolte $\mathrm{CH}$, Endres $\mathrm{M}$. Impact of prehospital triage scales to detect large vessel occlusion on resource utilization and time to treatment. Stroke 2018;49: 439-46.

25. Turc G, Maïer B, Naggara O, et al. Clinical scales do not reliably identify acute ischemic stroke patients with large-artery occlusion. Stroke 2016;47:1466-72.

26. Wendt $M$, Ebinger $M$, Kunz $A$, et al. Improved prehospital triage of patients with stroke in a specialized stroke ambulance: results of the pre-hospital acute neurological therapy and optimization of medical care in stroke study. Stroke 2015; 46:740-5.

27. Ebinger $M$, Winter $B$, Wendt $M$, et al. Effect of the use of ambulance-based thrombolysis on time to thrombolysis in acute ischemic stroke: a randomized clinical trial. JAMA 2014; 311:1622-31.

28. Southerland AM, Brandler ES. The cost-efficiency of mobile stroke units: Where the rubber meets the road. Neurology 2017;88:1300-1.

29. Cone DC, Cooley C, Ferguson J, et al. Observational multicenter study of a direct-to-CT protocol for EMS-transported patients with suspected stroke. Prehosp Emerg Care 2018;22: $1-6$.

30. Brinjikji W, Demchuk AM, Murad MH, et al. Neurons over nephrons: systematic review and meta-analysis of contrastinduced nephropathy in patients with acute stroke. Stroke 2017;48:1862-8.

31. Hughes S. PRISMS: no benefit of tPA in mild stroke without disability 2018 [Internet]. New York, NY: Medscape; 2018 [cited 2018 May 2]. Available from: https://www.medscape. com/viewarticle/892045.

32. Powers WJ, Rabinstein AA, Ackerson T, et al. 2018 Guidelines for the early management of patients with acute ischemic stroke: a guideline for healthcare professionals from the
American Heart Association/American Stroke Association. Stroke 2018;49:e46-110.

33. Vanacker $P$, Heldner MR, Amiguet $M$, et al. Prediction of large vessel occlusions in acute stroke: national institute of health stroke scale is hard to beat. Crit Care Med 2016;44:e336-43.

34. Kwah LK, Diong J. National Institutes of Health Stroke Scale (NIHSS). J Physiother 2014;60:61.

35. Martin-Schild S, Albright KC, Tanksley J, et al. Zero on the NIHSS does not equal the absence of stroke. Ann Emerg Med 2011;57:42-5.

36. Smith $E_{\text {, Kent } D M}$, Bulsara KR, et al. Accuracy of prediction instruments for diagnosing large vessel occlusion in individuals with suspected stroke: a systematic review for the 2018 guidelines for the early management of patients with acute ischemic stroke. Stroke 2018;49:e111-22.

37. Higgins P, MacFarlane PW, Dawson J, Mclnnes GT, Langhorne $P$, Lees KR. Noninvasive cardiac event monitoring to detect atrial fibrillation after ischemic stroke: a randomized, controlled trial. Stroke 2013;44:2525-31.

38. Song $D$, Lee $K$, Kim EH, et al. Value of utilizing both ASPECTS and CT angiography collateral score for outcome prediction in acute ischemic stroke. Int J Stroke 2015;10:1018-23.

39. Chalela JA, Kidwell CS, Nentwich LM, et al. Magnetic resonance imaging and computed tomography in emergency assessment of patients with suspected acute stroke: a prospective comparison. Lancet 2007;369:293-8.

40. Wardlaw JM, Seymour J, Cairns J, Keir S, Lewis S, Sandercock P. Immediate computed tomography scanning of acute stroke is cost-effective and improves quality of life. Stroke 2004;35: 2477-83.

41. Heldner MR, Hsieh K, Broeg-Morvay A, et al. Clinical prediction of large vessel occlusion in anterior circulation stroke: mission impossible? J Neurol 2016;263:1633-40.

42. Vymazal J, Rulseh AM, Keller J, Janouskova L. Comparison of CT and MR imaging in ischemic stroke. Insights Imaging 2012; 3:619-27.

43. Kidwell CS, Chalela JA, Saver JL, et al. Comparison of MRI and CT for detection of acute intracerebral hemorrhage. JAMA 2004;292:1823-30.

44. Coutts SB, Modi J, Patel SK, et al. CT/CT angiography and $\mathrm{MRI}$ findings predict recurrent stroke after transient ischemic attack and minor stroke: results of the prospective CATCH study. Stroke 2012;43:1013-7.

45. Sabarudin A, Subramaniam C, Sun Z. Cerebral CT angiography and CT perfusion in acute stroke detection: a systematic review of diagnostic value. Quant Imaging Med Surg 2014;4: 282-90. 


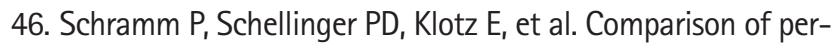
fusion computed tomography and computed tomography angiography source images with perfusion-weighted imaging and diffusion-weighted imaging in patients with acute stroke of less than 6 hours' duration. Stroke 2004;35:1652-8.

47. Ota T, Sato M, Amano T, Matsumaru Y. Door-to-needle time under 60 minutes and picture-to-puncture under 90 minutes: initiatives and outcomes in reducing time to recanalization for cerebral major artery occlusion. Neurol Med Chir (Tokyo) 2016;56:725-30.

48. Vemmos KN, Tsivgoulis G, Spengos K, et al. U-shaped relationship between mortality and admission blood pressure in patients with acute stroke. J Intern Med 2004;255:257-65.

49. Bowry R, Navalkele DD, Gonzales NR. Blood pressure management in stroke: five new things. Neurol Clin Pract 2014; 4:419-26.

50. McManus M, Liebeskind DS. Blood pressure in acute ischemic stroke. J Clin Neurol 2016;12:137-46.

51. Bath PM, Krishnan K. Interventions for deliberately altering blood pressure in acute stroke. Cochrane Database Syst Rev 2014:CD000039.

52. Lakshminarayan $\mathrm{K}$, Anderson DC, Borbas C, Duval S, Luepker RV. Blood pressure management in acute ischemic stroke. J Clin Hypertens (Greenwich) 2007;9:444-53.

53. Wu W, Huo X, Zhao X, et al. Relationship between blood pressure and outcomes in acute ischemic stroke patients administered lytic medication in the TIMS-China study. PLoS One 2016;11:e0144260.

54. Allison TA, Bowman S, Gulbis B, Hartman H, Schepcoff $S$, Lee K. Comparison of clevidipine and nicardipine for acute blood pressure reduction in patients with stroke. J Intensive Care Med 2017:885066617724340.

55. Stead LG, Gilmore RM, Vedula KC, Weaver AL, Decker WW, Brown RD Jr. Impact of acute blood pressure variability on ischemic stroke outcome. Neurology 2006;66:1878-81.

56. Huang $X$, Cheripelli BK, Lloyd SM, et al. Alteplase versus tenecteplase for thrombolysis after ischaemic stroke (ATTEST): a phase 2, randomised, open-label, blinded endpoint study. Lancet Neurol 2015;14:368-76.

57. Huang $X$, Maclsaac $R$, Thompson JL, et al. Tenecteplase versus alteplase in stroke thrombolysis: An individual patient data meta-analysis of randomized controlled trials. Int J Stroke 2016;11:534-43.

58. Campbell BC, Meretoja A, Donnan GA, Davis SM. Twenty-year history of the evolution of stroke thrombolysis with intravenous alteplase to reduce long-term disability. Stroke 2015; 46:2341-6.
59. Hacke W, Donnan G, Fieschi C, et al. Association of outcome with early stroke treatment: pooled analysis of ATLANTIS, ECASS, and NINDS rt-PA stroke trials. Lancet 2004;363:76874.

60. National Institute of Neurological Disorders and Stroke rt-PA Stroke Study Group. Tissue plasminogen activator for acute ischemic stroke. N Engl J Med 1995;333:1581-7.

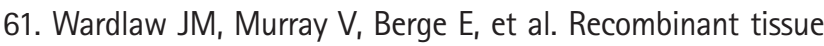
plasminogen activator for acute ischaemic stroke: an updated systematic review and meta-analysis. Lancet 2012;379: 2364-72.

62. Bluhmki $E_{1}$ Chamorro A, Davalos $A$, et al. Stroke treatment with alteplase given 3.0-4.5 h after onset of acute ischaemic stroke (ECASS III): additional outcomes and subgroup analysis of a randomised controlled trial. Lancet Neurol 2009;8: 1095-102.

63. You $S$, Saxena A, Wang $X$, et al. Efficacy and safety of intravenous recombinant tissue plasminogen activator in mild ischaemic stroke: a meta-analysis. Stroke Vasc Neurol 2018; 3:22-7.

64. Demaerschalk BM, Kleindorfer DO, Adeoye OM, et al. Scientific rationale for the inclusion and exclusion criteria for intravenous alteplase in acute ischemic stroke: a statement for healthcare professionals from the American Heart Association/American Stroke Association. Stroke 2016;47:581-641.

65. Fugate JE, Rabinstein AA. Absolute and relative contraindications to IV rt-PA for acute ischemic stroke. Neurohospitalist 2015;5:110-21.

66. Higashida R, Alberts MJ, Alexander DN, et al. Interactions within stroke systems of care: a policy statement from the American Heart Association/American Stroke Association. Stroke 2013;44:2961-84.

67. Banks JL, Marotta CA. Outcomes validity and reliability of the modified Rankin scale: implications for stroke clinical trials: a literature review and synthesis. Stroke 2007;38:1091-6.

68. Lansberg MG, Schrooten M, Bluhmki E, Thijs VN, Saver JL. Treatment time-specific number needed to treat estimates for tissue plasminogen activator therapy in acute stroke based on shifts over the entire range of the modified Rankin Scale. Stroke 2009;40:2079-84.

69. Saver JL, Gornbein J, Grotta J, et al. Number needed to treat to benefit and to harm for intravenous tissue plasminogen activator therapy in the 3- to 4.5-hour window: joint outcome table analysis of the ECASS 3 trial. Stroke 2009;40: 2433-7.

70. Schwamm LH, Wu O, Song SS, et al. Intravenous thrombolysis in unwitnessed stroke onset: MR WITNESS trial results. 
Ann Neurol 2018;83:980-93.

71. Thomalla G, Simonsen CZ, Boutitie F, et al. MRI-guided thrombolysis for stroke with unknown time of onset. N Engl J Med 2018:379:611-22.

72. Hussain $M$, Moussavi $M$, Korya $D$, et al. Systematic review and pooled analyses of recent neurointerventional randomized controlled trials: setting a new standard of care for acute ischemic stroke treatment after 20 years. Interv Neurol 2016; 5:39-50.

73. Saver JL, Gornbein J, Starkman S. Graphic reanalysis of the two NINDS-tPA trials confirms substantial treatment benefit. Stroke 2010;41:2381-90.

74. Khatri $P$, Tayama D, Cohen $G$, et al. Effect of intravenous recombinant tissue-type plasminogen activator in patients with mild stroke in the third international stroke trial-3: post hoc analysis. Stroke 2015;46:2325-7.

75. Khatri P, Kleindorfer DO, Devlin T, et al. Effect of alteplase vs aspirin on functional outcome for patients with acute ischemic stroke and minor nondisabling neurologic deficits: the prisms randomized clinical trial. JAMA 2018;320:156-66.

76. Behrouz R. Intravenous tenecteplase in acute ischemic stroke: an updated review. J Neurol 2014;261:1069-72.

77. Campbell BCV, Mitchell PJ, Churilov L, et al. Tenecteplase versus alteplase before thrombectomy for ischemic stroke. $\mathrm{N}$ Engl J Med 2018;378:1573-82.

78. Saver JL, Goyal M, Bonafe A, et al. Stent-retriever thrombectomy after intravenous t-PA vs. t-PA alone in stroke. N Engl J Med 2015;372:2285-95.

79. Nogueira RG, Jadhav AP, Haussen DC, et al. Thrombectomy 6 to 24 hours after stroke with a mismatch between deficit and infarct. N Engl J Med 2018;378:11-21.

80. Berkhemer OA, Fransen PS, Beumer D, et al. A randomized trial of intraarterial treatment for acute ischemic stroke. $\mathrm{N}$ Engl J Med 2015;372:11-20.

81. Campbell BC, Mitchell PJ, Kleinig TJ, et al. Endovascular therapy for ischemic stroke with perfusion-imaging selection. N Engl J Med 2015;372:1009-18.

82. Jovin TG, Chamorro A, Cobo E, et al. Thrombectomy within 8 hours after symptom onset in ischemic stroke. $\mathrm{N}$ Engl J Med 2015;372:2296-306.

83. Goyal M, Demchuk AM, Menon BK, et al. Randomized assessment of rapid endovascular treatment of ischemic stroke. N Engl J Med 2015;372:1019-30.

84. Goyal M, Menon BK, van Zwam WH, et al. Endovascular thrombectomy after large-vessel ischaemic stroke: a metaanalysis of individual patient data from five randomised trials. Lancet 2016;387:1723-31.
85. Bracard S, Ducrocq X, Mas JL, et al. Mechanical thrombectomy after intravenous alteplase versus alteplase alone after stroke (THRACE): a randomised controlled trial. Lancet Neurol 2016;15:1138-47.

86. Jung JM, Choi JY, Kim HJ, Seo WK. Statin use in spontaneous intracerebral hemorrhage: a systematic review and metaanalysis. Int J Stroke 2015;10 Suppl A100:10-7.

87. Rodrigues FB, Neves JB, Caldeira D, Ferro JM, Ferreira JJ, Costa J. Endovascular treatment versus medical care alone for ischaemic stroke: systematic review and meta-analysis. BMJ 2016;353:i1754.

88. Albers GW, Marks MP, Kemp S, et al. Thrombectomy for stroke at 6 to 16 hours with selection by perfusion imaging. $N$ Engl J Med 2018;378:708-18.

89. Sun $\mathrm{CH}$, Nogueira RG, Glenn BA, et al. "Picture to puncture": a novel time metric to enhance outcomes in patients transferred for endovascular reperfusion in acute ischemic stroke. Circulation 2013;127:1139-48.

90. Barlinn J, Gerber J, Barlinn K, et al. Acute endovascular treatment delivery to ischemic stroke patients transferred within a telestroke network: a retrospective observational study. Int J Stroke 2017;12:502-9.

91. Middleton S, Grimley R, Alexandrov AW. Triage, treatment, and transfer: evidence-based clinical practice recommendations and models of nursing care for the first 72 hours of admission to hospital for acute stroke. Stroke 2015;46:e1825.

92. Prabhakaran $\mathrm{S}$, Ward E, John $\mathrm{S}$, et al. Transfer delay is a major factor limiting the use of intra-arterial treatment in acute ischemic stroke. Stroke 2011;42:1626-30.

93. Froehler MT, Saver JL, Zaidat 00, et al. Interhospital transfer before thrombectomy is associated with delayed treatment and worse outcome in the STRATIS Registry (systematic evaluation of patients treated with neurothrombectomy devices for acute ischemic stroke). Circulation 2017;136:2311-21.

94. Fuentes $B$, Alonso de Lecinana $M$, Ximenez-Carrillo $A$, et al. Futile interhospital transfer for endovascular treatment in acute ischemic stroke: the madrid stroke network experience. Stroke 2015;46:2156-61.

95. Knoflach M, Lang W, Seyfang $L$, et al. Predictive value of $A B C D 2$ and $A B C D 3-I$ scores in TIA and minor stroke in the stroke unit setting. Neurology 2016;87:861-9.

96. Vora $N$, Tung CE, Mlynash $M$, et al. TIA triage in emergency department using acute MRI (TIA-TEAM): a feasibility and safety study. Int J Stroke 2015;10:343-7.

97. Zhang $\mathrm{Q}$, Wang $C$, Zheng $M$, et al. Aspirin plus clopidogrel as secondary prevention after stroke or transient ischemic at- 
tack: a systematic review and meta-analysis. Cerebrovasc Dis 2015;39:13-22.

98. Serebruany VL, Malinin Al, Ziai W, et al. Effects of clopidogrel and aspirin in combination versus aspirin alone on platelet activation and major receptor expression in patients after recent ischemic stroke: for the Plavix Use for Treatment of Stroke (PLUTO-Stroke) trial. Stroke 2005;36:2289-92.

99. Wang $Y$, Pan $Y$, Zhao $X$, et al. Clopidogrel with Aspirin in Acute Minor Stroke or Transient Ischemic Attack (CHANCE) trial: one-year outcomes. Circulation 2015;132:40-6.

100. McFarlane SI, Sica DA, Sowers JR. Stroke in patients with diabetes and hypertension. J Clin Hypertens (Greenwich) 2005; 7:286-92.

101. Glotzer TV, Ziegler PD. Cryptogenic stroke: is silent atrial fibrillation the culprit? Heart Rhythm 2015;12:234-41.

102. Chen JY, Zhang AD, Lu HY, Guo J, Wang FF, Li ZC. CHADS2 versus CHA2DS2-VASc score in assessing the stroke and thromboembolism risk stratification in patients with atrial fibrillation: a systematic review and meta-analysis. J Geriatr Cardiol 2013;10:258-66.

103. Kamel H, Okin PM, Elkind MS, ladecola C. Atrial fibrillation and mechanisms of stroke: time for a new model. Stroke 2016;47:895-900.

104. Melgaard L, Gorst-Rasmussen A, Lane DA, Rasmussen LH, Larsen TB, Lip GY. Assessment of the CHA2DS2-VASc score in predicting ischemic stroke, thromboembolism, and death in patients with heart failure with and without atrial fibrillation. JAMA 2015;314:1030-8.

105. Majule DN, Jing C, Rutahoile WM, Shonyela FS. The efficacy and safety of the WATCHMAN device in LAA occlusion in patients with non-valvular atrial fibrillation contraindicated to oral anticoagulation: a focused review. Ann Thorac Cardiovasc Surg 2018 Jun 29. https://doi.org/10.5761/atcs. ra.18-00014.

106. Kwong C, Ling AY, Crawford MH, Zhao SX, Shah NH. A clinical score for predicting atrial fibrillation in patients with cryptogenic stroke or transient ischemic attack. Cardiology 2017;138:133-40.

107. Finsterer J. Management of cryptogenic stroke. Acta Neurol Belg 2010;110:135-47.

108. Jaffre A, Ruidavets JB, Nasr N, Guidolin B, Ferrieres J, Larrue V. Tobacco use and cryptogenic stroke in young adults. J Stroke Cerebrovasc Dis 2015;24:2694-700.

109. Mojadidi MK, Mahmoud AN, Patel NK, Elgendy IY, Meier B. Cryptogenic stroke and patent foramen ovale: ready for prime time?. J Am Coll Cardiol 2018;72:1183-5.

110. Hutyra M, Pavlu L, Sanak D, et al. The role of echocardiogra- phy in patients after ischemic stroke. Cor Vasa 2016;58:e26172.

111. Morris JG, Duffis EJ, Fisher M. Cardiac workup of ischemic stroke: can we improve our diagnostic yield? Stroke 2009;40: 2893-8.

112. Lee MJ, Chung JW, Ahn MJ, et al. Hypercoagulability and mortality of patients with stroke and active cancer: the OASIS-CANCER study. J Stroke 2017;19:77-87.

113. Mackey J. Evaluation and management of stroke in young adults. Continuum (Minneap Minn) 2014;20(2 Cerebrovascular Disease):352-69.

114. Joundi RA, Martino R, Saposnik G, Giannakeas V, Fang J, Kapral MK. Predictors and outcomes of dysphagia screening after acute ischemic stroke. Stroke 2017;48:900-6.

115. Miles A, Zeng IS, McLauchlan H, Huckabee ML. Cough reflex testing in dysphagia following stroke: a randomized controlled trial. J Clin Med Res 2013;5:222-33.

116. Titsworth WL, Abram J, Fullerton A, et al. Prospective quality initiative to maximize dysphagia screening reduces hospitalacquired pneumonia prevalence in patients with stroke. Stroke 2013;44:3154-60.

117. Sorensen RT, Rasmussen RS, Overgaard $K$, Lerche $A$, Johansen $A M$, Lindhardt T. Dysphagia screening and intensified oral hygiene reduce pneumonia after stroke. J Neurosci Nurs 2013; 45:139-46.

118. Kalra L, Irshad S, Hodsoll J, et al. Prophylactic antibiotics after acute stroke for reducing pneumonia in patients with dysphagia (STROKE-INF): a prospective, cluster-randomised, open-label, masked endpoint, controlled clinical trial. Lancet 2015;386:1835-44.

119. Meader N, Moe-Byrne T, Llewellyn A, Mitchell AJ. Screening for poststroke major depression: a meta-analysis of diagnostic validity studies. J Neurol Neurosurg Psychiatry 2014;85: 198-206.

120. Beyaert C, Vasa R, Frykberg GE. Gait post-stroke: pathophysiology and rehabilitation strategies. Neurophysiol Clin 2015; 45:335-55.

121. Rand D, Zeilig G, Kizony R. Rehab-let: touchscreen tablet for self-training impaired dexterity post stroke: study protocol for a pilot randomized controlled trial. Trials 2015;16:277.

122. Rosenbaum T, Vadas D, Kalichman L. Sexual function in poststroke patients: considerations for rehabilitation. J Sex Med 2014;11:15-21.

123. Bembenek J, Karlinski M, Kobayashi A, Czlonkowska A. Early stroke-related deep venous thrombosis: risk factors and influence on outcome. J Thromb Thrombolysis 2011;32:96-102. 124. Pavon JM, Williams JW Jr, Adam SS, et al. Effectiveness of 
intermittent pneumatic compression devices for venous thromboembolism prophylaxis in high-risk surgical and medical patients [Internet]. Washington, DC: Department of Veterans Affairs; 2015 [cited 2018 May 2]. Available from: http://www. ncbi.nlm.nih.gov/books/NBK333230/.

125. CLOTS (Clots in Legs Or sTockings after Stroke) Trials Collaboration, Dennis $M$, Sandercock $P$, et al. Effectiveness of intermittent pneumatic compression in reduction of risk of deep vein thrombosis in patients who have had a stroke (CLOTS 3): a multicentre randomised controlled trial. Lancet 2013;382:516-24.

126. Martin D, Caso V, Kappelle J, et al. European Stroke Organisation (ESO) guidelines for prophylaxis for venous thromboembolism in immobile patients with acute ischaemic stroke. Eur Stroke J 2016;1:6-19.

127. Hemphill JC 3rd, Greenberg SM, Anderson CS, et al. Guidelines for the management of spontaneous intracerebral hemorrhage: a guideline for healthcare professionals from the American Heart Association/American Stroke Association. Stroke 2015;46:2032-60.

128. Qureshi Al, Palesch YY, Barsan WG, et al. Intensive blood-pressure lowering in patients with acute cerebral hemorrhage. $\mathrm{N}$ Engl J Med 2016;375:1033-43.

129. Anderson CS, Heeley E, Huang Y, et al. Rapid blood-pressure lowering in patients with acute intracerebral hemorrhage. $\mathrm{N}$ Engl J Med 2013;368:2355-65.

130. Le Roux P, Pollack CV Jr, Milan M, Schaefer A. Race against the clock: overcoming challenges in the management of anticoagulant-associated intracerebral hemorrhage. J Neurosurg 2014;121 Suppl:1-20.

131. Bechtel BF, Nunez TC, Lyon JA, Cotton BA, Barrett TW. Treat- ments for reversing warfarin anticoagulation in patients with acute intracranial hemorrhage: a structured literature review. Int J Emerg Med 2011;4:40.

132. Gregson BA, Rowan EN, Francis R, et al. Surgical Trial In Traumatic intraCerebral Haemorrhage (STITCH): a randomised controlled trial of Early Surgery compared with Initial Conservative Treatment. Health Technol Assess 2015;19:1-138.

133. Jeong HG, Kim BJ, Yang MH, Han MK, Bae HJ, Lee SH. Stroke outcomes with use of antithrombotics within 24 hours after recanalization treatment. Neurology 2016;87:996-1002.

134. Amin A, Deitelzweig $S$, Jing $Y$, et al. Estimation of the impact of warfarin's time-in-therapeutic range on stroke and major bleeding rates and its influence on the medical cost avoidance associated with novel oral anticoagulant use-learnings from ARISTOTLE, ROCKET-AF, and RE-LY trials. J Thromb Thrombolysis 2014; 38:150-9.

135. Patel MR, Mahaffey KW, Garg J, et al. Rivaroxaban versus warfarin in nonvalvular atrial fibrillation. N Engl J Med 2011; 365:883-91.

136. Keating GM. Apixaban: a review of its use for reducing the risk of stroke and systemic embolism in patients with nonvalvular atrial fibrillation. Drugs 2013;73:825-43.

137. Hart RG, Pearce LA, Aguilar MI. Meta-analysis: antithrombotic therapy to prevent stroke in patients who have nonvalvular atrial fibrillation. Ann Intern Med 2007;146:857-67.

138. Ezekowitz MD, Bridgers SL, James $K E$, et al. Warfarin in the prevention of stroke associated with nonrheumatic atrial fibrillation. Veterans Affairs Stroke Prevention in Nonrheumatic Atrial Fibrillation Investigators. N Engl J Med 1992; 327:1406-12. 